\title{
Conflits Armés Et Fragilité De L'autorité Étatique Au Nord-Kivu En République Démocratique Du Congo
}

\author{
Tabin Lissendja Bahama \\ Politologue, Enseignant-Chercheur, Chef de Travaux, \\ Université de Kisangani, République Démocratique du Congo
}

doi: 10.19044/esj.2017.v13n5p457 URL:http://dx.doi.org/10.19044/esj.2017.v13n5p457

\begin{abstract}
This article analyzes the official institutional weakness of Congo's state in its quest to strengthen its authority in the province of North Kivu. Indeed, since 1998, it became obvious that state authority at both the central and provincial levels in the Democratic Republic of the Congo (DRC) were weakened by armed groups' activism, war-based economy, borders' looseness, militarization of local economy, and its extraversion. The most obvious challenge today is that private actors and organizations are acting in networks and mobilizing strategies. These strategies were employed in order to prevent the expansion of state authority over their areas of intervention. The presence of the latter would heavily affect the emergence and the pursuit of business, often associated with illicit practices. The operatory mode of this predation considerably limits Congo's state capacity and it was to the detriment of the local population. This study revealed that Congo's state is no longer capable of redeploying its authority in the province of North Kivu. On the other hand, it does not want to do it anymore because this state of chaos paradoxically protects the political and economic interests of certain members of the ruling class. Its apparent weakness denotes more unwillingness than a real incapacity.
\end{abstract}

Keywords: Fragile state, state authority, armed groups, predation, war economy

\section{Résumé}

La présente réflexion se propose d'analyser l'incapacité officielle de l'Etat congolais à assoir son autorité dans la province du Nord-Kivu à l'Est de la République Démocratique du Congo. En effet, il s’observe depuis les années 1998 que le pouvoir politique tant central que provincial en RDC se trouve affaibli par l'activisme des groupes armés, la mise en place d'une économie de guerre, la perméabilité des frontières, la militarisation de 
l'économie locale et son extraversion vers l'extérieur. Le constat amer est que les acteurs et organisations privés s'organisent pour limiter le déploiement de l'autorité de l'Etat dans leurs zones d'intervention afin d'empêcher que sa présence nuise considérablement à l'émergence et à la poursuite de leurs activités, souvent criminelles. Le mode opératoire de cette prédation limite considérablement la capacité de l’Etat congolais et ce, au détriment de la population locale qui paie le lourd tribut de l'activisme des acteurs de la violence. L'Etat congolais se voit incapable de redéployer son autorité au Nord-Kivu ou, mieux, ne veut pas le faire afin de protéger les avantages politiques et économiques de certains dirigeants étatiques. Sa faiblesse constaté dénote plus d'un non vouloir que d'une incapacité réelle. Les populations locales du Nord-Kivu continueront de se battre contre les acteurs locaux, nationaux, régionaux et internationaux qui cherchent à protéger leurs intérêts dans leur région. Tant que les ressources naturelles seront disponibles et accessibles au moyen de la violence, les populations du Nord-Kivu risquent de passer leur vie dans un contexte de violence permanente et risqueraient d'être massacrées en essayant de protéger leurs ressources.

Mots clés : Etat fragile, autorité étatique, groupes armés, prédation, économie de guerre

\section{Introduction}

La récurrence des conflits armés au Nord-Kivu impacte négativement sur l'environnement politique, sécuritaire, économique et social de l'ensemble de la Province du Nord-Kivu. De façon logique, c'est l'Etat, en tant que structure d'organisation, de régulation, de contrôle, d'arbitre de la société qui est mis en épreuve devant les différentes forces sociales internes et externes. Devant la contestation manifeste de son autorité par des personnes et organisations privées, tout Etat se voulant respectueux et responsable se doit de rétablir la situation par le déploiement de son autorité sur les parties du territoire qui cherchent à échapper ou se soustraire de son contrôle. Cette capacité de faire face aux tentatives de privatisation de la violence au sein de l'Etat par des groupes particuliers n'est pas l'apanage de tout Etat. Certains en souffrent durablement au risque de parvenir à l'affaiblissement considérable de leurs capacités d'intervention. L'Afrique en fourni des exemples éloquents.

Certains auteurs considèrent que la conception wébérienne de l'Etat fait largement défaut parce que le principe de monopole de la violence légitime, de même que celle de l'étanchéité des sphères publiques et privées, ne correspondent pas à la réalité de la plupart des Etats africains. En d'autres termes, le fossé entre la norme wébérienne et la réalité africaine ne peut être 
qu’énorme. Deux grandes explications, liées l'une à l'autre, se dessinent alors : soit que les représentants de l'Etat sont «incapables » de diffuser l'autorité étatique à un degré qui s’apparenterait au modèle wébérien, soit qu'ils ne le « veulent pas». Pouvoir et vouloir appellent ici à des explications distinctes » (Jourde C, 2009). Dans le premier cas de figure, les élites étatiques n’ont pas les moyens institutionnels, économiques, et/ou discursifs de s’imposer aux forces politiques qui s’opposent à l'idée de l'Etat wébérien. Dans le second cas de figure, le problème n'en est pas un d'incapacité, mais plutôt de non vouloir. Les élites étatiques africaines refusent ici consciemment de procéder à une projection soutenue de l'autorité étatique. Ce refus de jouer le jeu étatique s'expliquerait soit parce que ces élites étatiques sont elles-mêmes issues des communautés, classes sociales ou réseaux qui s’opposent à une présence trop forte de l'Etat, ou alors parce qu'elles profitent politiquement et économiquement de la faiblesse de l'Etat (Jourde C, 2009).

En plus de la grille d'analyse présentée par Cédric Jourde, Achille Mbembe (2000) estime qu'il faudrait ajouter une autre variable d'analyse, à savoir les calculs et stratégies des représentants de l'Etat. Dans un contexte de libéralisation de l'économie mondiale et de la pression des institutions financières internationales, les représentants étatiques se seraient adaptés de manière rationnelle à ces contraintes en produisant une forme différente d’autorité étatique, le «gouvernement privé indirect». Ainsi, les représentants de l'Etat opèrent un retrait stratégique préférant déléguer (et non pas délaisser) à des acteurs privés ou semi-privés des fonctions normalement perçues comme régaliennes. Les élites étatiques africaines sont donc engagées dans un processus de privatisation, une politique de « décharge » d’activités publiques à la faveur d’acteurs privés. Pour William Reno (1999), le processus irait plus encore loin, dans la mesure où, dans plusieurs pays africains, les élites politiques vont jusqu’à accélérer le démantèlement de l'Etat, de manière rationnelle et intentionnelle.

Telle semble être la situation de plusieurs pays d'Afrique confrontés aux différents facteurs internes et externes de contestation de leurs autorités. Dans certains cas, ce sont les détenteurs du pouvoir qui sont contestés par les groupes particuliers et s'organisent, aux moyens de la violence, pour chercher à les remplacer. Mais dans d'autres circonstances, c'est le principe même de l'Etat qui est remis en cause, les groupes particuliers cherchant à limiter considérablement son pouvoir en vue de disposer des espaces d'actions élargie pour la mise en œuvre de leurs activités, souvent illicites et criminelles. La République Démocratique du Congo, à l'instar des pays d'Afrique, est confrontée à la problématique de la faiblesse de l'Etat à assurer ses responsabilités, mêmes régaliennes, face aux forces de tous ordres qui lui contestent sa prééminence politique et son monopole de la 
contrainte légitime. La manifestation de la faiblesse de l'Etat s’est accentuée avec les conflits armés qui ont secoué et secouent encore le pays, particulièrement dans sa partie Est. La situation d'insécurité permanente et de l'inefficacité de l'Etat et de ses services dans des vastes zones instables de la province du Nord-Kivu appellent des analyses approfondies des chercheurs afin de faire l’éclairage sur le déroulement des événements sur le terrain.

Les préoccupations suivantes sont au centre de la présente réflexion scientifique : pourquoi l'autorité étatique apparait-il fragile dans la province du Nord-Kivu ? L’Etat congolais est-il incapable de déployer son autorité sur l'ensemble de la province du Nord-Kivu ? Comment se présentent les effets de la fragilité de l'Etat sur l'environnement politique, économique, social et sécuritaire du Nord-Kivu ? Il est aisé de postuler que la fragilité de l'autorité étatique au Nord-Kivu serait consécutive à l'existence et à l'activisme des forces internes et externes qui lui sont supérieures et l'empêche, d'une certaine manière, de remplir ses fonctions régaliennes. Par ailleurs, il s'observe que l'Etat congolais ne serait pas incapable de déployer son autorité sur l'ensemble de la province mais se réserverait de le faire efficacement au risque de nuire aux intérêts économiques et politiques dont certains acteurs étatiques seraient impliqués et en tireraient énormément des profits divers. La fragilité de l'autorité étatique se manifesterait à travers le difficile contrôle du pouvoir central sur la province du Nord-Kivu, le difficile contrôle du pouvoir provincial sur les territoires, le recours systématique à la violence comme mode d'expression politique par les acteurs concernés et la porosité des frontières entre le Nord-Kivu et les pays voisins de l'Est, le Rwanda et l'Ouganda en particulier.

\section{Matériels et méthodes}

Les stratégies mises en place par les acteurs pour construire la réalité macabre de la province du Nord-Kivu sont au centre de la présente réflexion. Elle est ainsi développée dans une perspective constructiviste qui consiste à déconstruire les réalités apparentes, œuvre d’acteurs intentionnels, afin de saisir les faits dans leurs profondeurs réelles et leurs réalités premières. Les données ici présentées ont été récoltés par entretien dans les principales villes du Nord-Kivu qui sont Goma, Butembo et Beni ainsi que les territoires de la province qui sont Beni et Lubero (Grand-Nord) ainsi que Rutshuru, Nyiragongo, Masisi, Walikale (Petit-Nord). Notre entretien est donc semidirectif dans la mesure où il s’agit, selon J. Preyssinet-Dominjon (1997), d'une «technique adaptée à la compréhension en profondeur des phénomènes complexes de la société : les sujets livrent leur conception de la réalité, leurs visions du monde, leur système de valeurs ou de croyance, le sens qu'ils attribuent aux objets ou aux comportements ». 
Cet entretien semi-directif a été réalisé avec un minimum de structuration, axé sur des thèmes ouverts avec liberté d'expression du répondant. Dans cette optique, le choix des sujets à interroger dépendait de nous-mêmes et ce, en fonction de ce qu'on attendait d'eux et compte tenu de leur capacité à saisir la pertinence et la complexité de l’objet de notre étude. Ainsi, les entretiens ont été réalisés avec 40 sujets dont 18 dans le Grand Nord et 22 dans le Petit Nord. Parmi les personnes interviewées, il y a les combattants de groupes armés, les acteurs politiques, les opérateurs économiques, les leaders communautaires et les acteurs de la société civile du Nord-Kivu. Ces données ont été récoltées pendant la période allant de 2013 et 2015. Les contenus des informations récoltées ont été scientifiquement analysés à travers la technique d’analyse qualitative de contenu pour être présentées dans le cadre de cette réflexion.

\section{Résultats}

\section{Environnement socio-économique du Nord-Kivu}

Le conflit congolais est souvent perçu et analysé comme une guerre de prédation perpétrée par différents acteurs. Il est connu que tout conflit armé entraine de conséquences positives et négatives sur le lieu de son déroulement et ce, dans plusieurs domaine de la vie des membres qui y vivent. Dans cette présentation des résultats de l'étude, il sera question d'analyser les conséquences sociales et économiques de la persistance des conflits armés dans la province du Nord-Kivu, facteurs qui contribuent à l’affaiblissement et à la fragilité de l'autorité étatique dans la province.

\section{L’économie de guerre au Nord-Kivu}

Pour Philippe le Billon (2003), « les rapports entre guerres et ressources naturelles ont conduit à une écologie politique de la guerre analysant les guerres de ressources, environnementales, de pillage ou de sécession liées aux ressources naturelles. Un État détenteur de ressources en hydrocarbures a neuf fois plus de risques d'être le théâtre de conflits armés qu'un État non pourvu. Les ressources naturelles peuvent fournir les moyens de financer les rébellions motivées par d'autres intérêts que les ressources elles-mêmes. Les ressources naturelles du sous- sol peuvent, du fait de leur abondance, attiser des contrôles par la violence (coloniale, impérialiste, pillage). Les ressources naturelles du sol peuvent, du fait de leur rareté (eau, terre), aviver les tensions ». La guerre doit être financée et elle peut avoir des motivations de captation de richesses. Certains travaux économétriques décomposent les facteurs explicatifs alors que d'autres utilisent la méthode systémique (Philippe Hugo, 2009).

De nombreux travaux montrent le rôle spécifique des ressources naturelles dans la conflictualité. En 2004, Collier et Hoeffler (2012) 
analysent cinquante-deux guerres civiles entre 1960 et 1999. Ils montrent que la dépendance économique envers les ressources naturelles augmente les risques de guerre civile pendant les cinq années qui suivent l'amplification de cette dépendance. De même Fearon et Laitin (1997) démontrent que le risque de guerre civile dans un pays exportateur de pétrole est de 19,1\% contre 9,2\% pour un pays non doté en ressources pétrolières. Reynal-Querol (2002) analyse le lien entre l'abondance de ressources naturelles et l'occurrence de conflits civils interethniques. Pour 138 pays entre 1960 et 1995, l'abondance en ressources naturelles est un des facteurs essentiels. Enfin, Doyle et Sambanis (2000) montrent que les guerres civiles sont plus longues pour les pays dotés en ressources naturelles. Dans la typologie des conflits liés aux ressources naturelles, on peut différencier le rôle des ressources naturelles selon le double critère de concentration et d'espace (filières nationales et internationales). Le tableau qui suit donne une lumière de ce dont il s’agit.

Tableau 1 : Schématisation de guerres pour le contrôle des ressources.

\begin{tabular}{|c|c|c|}
\hline Filières & Concentration territoriale & Dissémination \\
\hline International/régional & $\begin{array}{c}\text { Guerres énergétiques : } \\
\text { hydrocarbures. } \\
\text { Etat rentiers et investissements } \\
\text { lourds ; Liens oligopoles/Appareil } \\
\text { d’Etat ; Opposition } \\
\text { gouvernement/rebelles ; Enjeux } \\
\text { de puissances ; Sécession ou } \\
\text { autonomie (Casamance, Ogaden, } \\
\text { Delta du Niger) ; (Angola, } \\
\text { Congo, Niger, Nigeria, Soudan, } \\
\text { Tchad }\end{array}$ & $\begin{array}{c}\text { Guerre de pillage minier, } \\
\text { filière artisanale : diamant, } \\
\text { Coltan, Or. } \\
\text { Economie de guerre } \\
\text { militarisée, main d'œuvre } \\
\text { élevée, armées et milices à } \\
\text { base ethnique et nationale. } \\
\text { Rôle de réseaux Liberia, Sierra } \\
\text { Léone, RDC (Kivu, Katanga) }\end{array}$ \\
\hline National/local & $\begin{array}{l}\text { Guerres environnementales : } \\
\text { ressources rares du sol : eau, } \\
\text { terres arables, forêts, pâturages, } \\
\text { allogènes/autochtones. Milices à } \\
\text { base régionale : Darfour, Côte } \\
\text { d’Ivoire, Rwanda }\end{array}$ & $\begin{array}{c}\text { Guerre de trafic, commerce } \\
\text { illicite : drogue, rançon. } \\
\text { Seigneur de guerre, réseaux } \\
\text { terroristes. } \\
\text { Economie de marché de } \\
\text { guerre ; } \\
\text { Etat failli (non-droit, fiscalité, } \\
\text { douane, contrôle terroriste). } \\
\text { Ex : Somalie. }\end{array}$ \\
\hline
\end{tabular}

Source : Philippe Hugo « Le rôle des ressources naturelles dans les conflits en Afrique », La découverte n¹34, mars 2009, pp. 63-79

Les conflits sont, selon des degrés différents, une combinaison des quatre configurations. Tel est le cas au Kivu où il y a contrôle des mines de coltan, d'or, d'étain par les rebelles hutus, tutsis, les troupes de Kabila ou de Kagamé comme l'a bien souligné Jacquemot (2009). Au Nord-Kivu, une économie militaire et criminelle s’organise autour des filières du coltan, de 
l'or et de l'étain mais aussi de diamant. Ces filières ont elles-mêmes des ramifications régionales et internationales. Comme indiqué dans le tableau élaboré par Philippe Hugo, les conflits armés au Nord-Kivu est à la fois une guerre environnementale du fait de la question de l'accès à la terre, mais elle a été amplifiée, régionalisée et globalisée par différents acteurs en quête des ressources naturelles congolaises indispensables au fonctionnement et à la survie des industries de transformation européenne, asiatique et américaine. La question environnementale pouvait bien être réglée sur le plan local ou national si jamais les intérêts économiques des acteurs globaux n'en étaient pas liés. Les questions de la justice, des droits de l'homme, de la violence sexuelle, de l'ethnicité ou de la gouvernance sont malheureusement supplantées et englouties par des préoccupations économiques des acteurs.

En effet, les deux guerres de 1996 et 1998 sont venues aggraver la situation économique du pays, « en entraînant la destruction de nombreuses infrastructures et en généralisant les pratiques de pillage des ressources héritées du régime Mobutu sous couvert de financer l'effort de guerre. "S'il n'y avait pas eu de clients désireux de faire le commerce de ces ressources », il n'y a en effet jamais eu pénurie d'acheteurs étrangers prêts à faire le commerce de ces marchandises en dépit de l'existence de rapports dénonçant les graves violations du droit international commises par leurs partenaires commerciaux et financiers. Les acheteurs sont non seulement des négociants en RDC et dans les pays voisins, mais aussi des sociétés privées enregistrées dans d'autres pays, y compris des sociétés multinationales. "Dans certains cas, les sociétés multinationales participaient directement aux négociations avec les auteurs de violations des droits de l'homme, payaient des groupes armés ou leur fournissaient des installations ou des moyens logistiques pour la prédation des ressources naturelles » (Rapport du groupe d'expert de l'ONU, 2008).

Cette permanence de l'insécurité, de la violence et de la guerre au Nord-Kivu liée à l'accès violent aux ressources naturelles a ainsi crée de conditions favorables à l'émergence de plusieurs autres activités criminelles. Il s'agit des assassinats, de tueries, de braconnage, de destruction forestière, de coupure de routes, d'enlèvement accompagné de demande de rançon, de pillage, de vol, de viol, de travaux forcés proche de l'esclavage,...Toutes ces activités ont trouvé du champ libre et se développent du fait de l'absence de la réglementation. La guerre, qui constitue une contestation flagrante de l'autorité de l'Etat, paralyse tous les systèmes de sécurité et de contrôle de la société par les services publics. Toutes les activités criminelles ci-haut énumérées sont solidement installées au Nord-Kivu et se réalisent en toute impunité. Les auteurs de ces activités se retranchent tranquillement dans les zones difficile d'accès où l'Etat congolais ne peut pas ou ne veut pas aller les 
chercher au regard de coûts humains, logistiques et financiers que l'engagement pourrait entrainer.

\section{Militarisation de l'économie locale}

La province du Nord-Kivu est celle qui regorge à ce jour le nombre le plus élevé de militaires congolais en comparaison avec les autres provinces du pays. Leur objectif principal est de protéger les frontières nationales et de sécuriser les populations locales, bien qu'ils y parviennent difficilement ou qu'ils ne veulent pas le faire correctement. Sur cet effectif de l'armée, il convient d'ajouter les combattants de différents groupes armés qui sévissent dans la province. Il en résulte un nombre très élevé d’hommes en armes qui, souvent, débordent les frontières de leurs compétences pour s’ingérer dans d'autres domaines de la vie pour des raisons de survie, de protection des personnes ou d'affaires personnelles. Par militarisation de l'économie ici, nous attendons l'implication significative des acteurs militaires ou hommes en armes dans les activités économiques d'un milieu déterminé. En RDC, particulièrement dans sa partie Est, les militaires et groupes armés nationaux et étrangers sont directement ou indirectement impliqués dans les activités économiques qui émergent dans la région du fait de la guerre.

Cette nouvelle configuration de l'implication des hommes en armes dans le circuit économique au Nord-Kivu qui était, jadis essentiellement aux mains des civiles, a fortement reconfiguré le paysage économique de la province et redessiner les cartes et jeu d'alliance parmi les acteurs impliqués, militaires ou civils. Les plus faibles ont fini par fuir la province pour développer leurs activités économiques ailleurs ou ont fini par faire purement et simplement faillite. Par contre, les plus entreprenants acteurs économiques ont dû développer des capacités d’adaptation avec la nouvelle configuration économique transformée par l'arrivée en scène des acteurs armés. Pour se protéger et protéger leurs affaires, les acteurs économiques du Nord-Kivu ont dû faire des alliances avec les acteurs armés en vue de bénéficier des avantages nécessaires à la survie de leurs activités économiques.

La croissance des chiffres d'affaires des opérateurs économiques de la province, dont la plupart évolue dans le secteur minier, peut s’expliquer de plusieurs manières. D’abord, la majorité d'opérateurs économiques de la province contourne le paiement des fiscs par le truchement de leurs coopérants militaires soit de l'armée congolaise, soit des groupes armés nationaux et étrangers, soit des officiels politiques de la province ou du niveau national. En contrepartie, les opérateurs économiques concernés versent des sommes d'argent à leurs protecteurs en guise de récompense afin 
de consolider les liens et maintenir le climat d'affaires ${ }^{61}$. Ainsi, chaque opérateur économique fait effort pour disposer d'une protection occulte des hommes en armes pouvant intervenir si nécessaire pour la protection de ses affaires. Ensuite, les acteurs armés, appartenant soit aux FARDC soit aux groupes armés nationaux et étrangers, deviennent rapidement et illégalement détenteurs des capitaux qu'ils placent ou investissent dans les affaires économiques de la province. Leurs capitaux sont ainsi tenus par les acteurs civils qui travaillent pour leur compte tout en tirant de bénéfices à leur niveau. Les activités économiques des acteurs civils officiels sont ainsi protégées et suivies par les acteurs armés occultes mais véritables propriétaires des capitaux ${ }^{62}$. D’autres opérateurs économiques travaillent directement avec les capitaux étrangers ou des courtiers positionnés dans les pays voisins de l'Est et leurs activités économiques sont également protégées par les acteurs armés sur le terrain avec lesquels ils coopèrent étroitement.

Enfin, la corruption qui bat son plein dans la province favorise l'émergence et la prospérité des activités illicites et frauduleuses. Les opérateurs économiques, capitalistes par excellence, préfèrent mieux traiter avec les petits agents de l'Etat à qui ils disposent de petites sommes d'argent (pot de vin) plutôt que d'encourager le retour à l'institutionnalisation et la réhabilitation de services de l'Etat. Ils seraient donc les premiers à soutenir les groupes armés existants ou à entretenir des nouveaux pour le contrôle des zones d'exploitation ou des zones frontalières, stratégie efficace à la prospérité de leurs activités économiques dans la province. Cette stratégie des acteurs économiques du Nord-Kivu de coopérer avec les acteurs armés pour l'émergence de leurs activités économiques constitue un énorme manque à gagner pour l'Etat congolais en termes d'impôts réel à verser dans le trésor public. Ceci traduit également, d'une part, l’incapacité de l'Etat congolais à imposer un contrôle total sur son territoire du fait des actions des acteurs privés qui lui opposent une résistance véritable, acteurs privés ayant de ramification avec d'autres acteurs régionaux et internationaux qui résistent aux efforts de l'Etat à imposer son autorité. D'autre part, cette réalité traduirait également une certaine complicité de l'Etat congolais qui laisserait délibérément du champ libre aux acteurs privés pour mener des actions militaires et économique dont certains membres du gouvernement tireraient des bénéfices économiques ou politiques.

\section{Extraversion économique du Nord-Kivu vers l'Afrique de l'Est}

\footnotetext{
${ }^{61}$ Entretien avec les membres de DGDA à la frontière congolaise de Bunagana dans le territoire de Rutshuru en Province du Nord-Kivu, octobre 2012.

${ }^{62}$ Entretien avec les membres de la DGDA à la frontière congolaise de Kasindi situé à 86 kilomètres de la ville de Beni dans le territoire de Beni au Nord-Kivu, novembre 2012.
} 
Depuis la guerre de 1996 sous la conduite de l’AFDL, les activités économiques au Nord-Kivu semble s'orienter vers les pays voisins de l'Est jusqu'aux pays asiatiques que sont la Chine, l'Inde, les Emirats Arabes Unis et autres. Cette extraversion économique s'était accentuée avec la guerre du RCD et la longue rupture politique entre Goma et Kinshasa. Cette rupture avait permis aux autorités politiques et militaires du Nord-Kivu de vivre l'expérience d'une gouvernance autonome bien que sous l'emprise des pays voisins, le Rwanda et l'Ouganda en particulier. Sur le plan économique, il porte à croire que les opérateurs économiques, familiarisés avec les opérations illicites, la fraude et le gain facile, auraient eu du mal à supporter le contrôle fiscal et la tendance à la restauration de la légalité dans les activités économiques de la province. Cette volonté politique du gouvernement central s'est heurtée à une résistance permanente des acteurs économiques du milieu.

Dans la même optique, Roland Pouriter (2009) note : «Eloignés de plus de $1500 \mathrm{~km}$ à vol d'oiseau de la capitale, le Nord et le Sud-Kivu entretiennent peu de relations économiques avec l'ouest du Congo. Les échanges sont tournés vers l'Afrique de l'Est et l’Océan Indien. La guerre civile a, par la force des choses, renforcé les tendances centrifuges d'une périphérie coupée de l'hinterland congolais. Les programmes de reconstruction des infrastructures de communication ne sont pas suffisamment avancés pour que le Kivu soit à nouveau ancré à l'espace économique congolais ». Dans l'émergence et la sécurisation de leurs activités, les opérateurs économiques du Nord-Kivu préfèrent garder contact et maintenir le lien avec les pays voisins de l'Est d'où sont installés les grands comptoirs d'achat de minerais en destination des pays occidentaux et asiatiques. En effet, il est bien connu qu'avant le déclenchement des guerres en RDC, ce sont les activités agro-pastorales qui constituaient le symbole de la supériorité économique des éleveurs tutsis par rapport aux agriculteurs Hunde, Tembo, Nyanga, Hutu dans le Petit Nord. Mais avec les rebellions de l'AFDL puis celle du RCD ayant occasionné la course vers les minerais exploités de façon artisanale mais surtout illicite, les activités agro-pastorales ont cédé place à l'exploitation artisanale des minerais, le coltan et la cassitérite en particulier.

L'évacuation des minerais vers les pays de l'Est a également ouvert la voie de l'Est pour l'approvisionnement en produits de première nécessité pour ravitailler les travailleurs dans les sites d'exploitation des minerais. Les échanges se sont ainsi intensifiés jusqu'à devenir permanents entre les opérateurs économiques du Nord-Kivu et ceux des pays voisins pour l'achat et la vente des produits de première nécessité et pour les produits de base. Pendant tout le temps de la guerre, et bien longtemps après, les opérateurs économiques du Nord-Kivu ont maintenu les échanges commerciaux avec 
les pays de l’Est et de l’Asie jusqu’à ce jour. En dépit de la réunification du pays et le redéploiement de l'Etat et de ses services sur l'ensemble du territoire national, les activités économiques du Nord-Kivu restent orientées vers les pays de l'Est et de l'Asie au détriment de Kinshasa et des pays occidentaux.

Deux autres facteurs importants consolident l'extraversion économique du Nord-Kivu vers les pays de l'Est et de l'Océan indien : la proximité et le coût de transport pour atteindre les zones d'approvisionnement. En ce qui concerne la proximité, il convient de préciser que les opérateurs économiques, par nature capitalistes, font toujours les calculs coûts-intérêts dans leurs opérations économiques. La proximité d'atteindre les pays d'approvisionnement à partir de Goma est évidente par rapport à Kinshasa. Pour se rendre en Europe, en Asie ou en Afrique du Sud, il est quasi facile de prendre le transport routier de Goma à Kigali ou de Goma à Kampala afin de prendre le vol international et se rendre à la destination choisie, plutôt que de prendre l'option de passer par Kinshasa, déjà très éloigné de la ville de Goma avec toutes les tracasseries administratives et policières connues de tous. Pour ce qui est du coût de transport, le déplacement des personnes et des biens coûtent moins cher depuis Goma pour se rendre à l'Est de l'Afrique, en Europe et en Asie en comparaison d'un départ programmé depuis Kinshasa.

Par ailleurs, il convient également de dire que cette comparaison en termes de proximité et de coût de transport attirent déjà quelques opérateurs économiques de l'Ouest qui préfèrent désormais passer par l'Est pour l'approvisionnement de leurs marchandises depuis l’Océan indien. Au fait, l'impulsion a été donnée par les opérateurs économiques de l'Est qui ont ouvert les activités économiques à Kinshasa, les Nande surtout, qui maintiennent leurs sources d'approvisionnement des produits jusqu'à Kinshasa. Pour arriver à Kinshasa, les marchandises suivent d'abord les mêmes trajets (Chine, Japon, Inde, Dubaï) jusqu’à Mombasa ou Dar-esSalam pour arriver à Goma ou Butembo en passant par Kampala ou Kigali jusqu'aux frontières congolaises (Goma, Bunagana, Kasindi). A partir de Goma ou Butembo, les véhicules chargent les marchandises jusqu’à Kisangani pour enfin prendre les bateaux jusqu’à Kinshasa. En termes de coût de transport, la voie de l'Est demeure moins couteuse que de faire partir les marchandises des pays de l'Océan indien jusqu'au port de Matadi en contournant l'Afrique du Sud.

Il a fallu attendre la fin du monopole de transport aérien par CAA en 2015 par le démarrage de la compagnie nationale «Congo Airways » pour que les coûts de transport entre Goma et Kinshasa soient revu à la baisse (260 dollars américains contre 450 dans le passé: aller-simple). Mais en dépit de cet événement, les circuits économiques sont restés tournés vers l’Est de 
l'Afrique et l'Océan Indien non seulement pour les opérateurs économiques du Nord-Kivu mais aussi pour ceux de Kinshasa qui ont trouvé utile de suivre cette voie. Cette tendance est encore renforcée par l'ouverture de l'aéroport de Goma aux trafics internationaux. La poursuite de la prédation de ressources naturelles par les groupes armés au Nord-Kivu et leur évacuation vers les pays de l'Est favorisent et confortent cette extraversion économique. Il faudra beaucoup d'effort et de volonté politique pour que Kinshasa renverse la tendance.

\section{Discussion}

\section{L'environnement politique et sécuritaire au Nord-Kivu}

L'observation rigoureuse de l'univers politique congolais laisse entrevoir que depuis le déclenchement de la guerre de l'Alliance de Forces Démocratique pour la Libération du Congo (AFDL), la Province du NordKivu se retrouve dans une situation bien particulière. Point de départ des mouvements insurrectionnels et bastion ou zones de retranchement de groupes armés nationaux et étrangers, le Nord-Kivu est désormais au centre des intérêts de mouvements et groupes particuliers. La poursuite de la guerre à travers la rébellion du Rassemblement Congolais pour la Démocratie (RCD) et les groupes armés nationaux et étrangers au Nord-Kivu met l'Etat congolais devant une dure épreuve liée à son existence et ses capacités en tant que structure de régulation d'actions individuelles et collectives. De plus, la montée en puissance de l'insécurité et la poursuite des conflits armés limitent systématiquement l'efficacité des actions de l'Etat et lui fait perdre toute confiance dans le chef de la population locale. Il s'observe que le NordKivu semble progressivement échapper au contrôle politique et sécuritaire de l'Etat congolais, à travers les actions d'acteurs privés, et se rend incapable de remplir ses fonctions régaliennes.

\section{Difficile contrôle du pouvoir central sur le Nord-Kivu}

Depuis 1996, l'année de l'entrée en force de l'AFDL au Zaïre à travers son action insurrectionnelle, le Nord-Kivu semble être pris au piège par différents acteurs impliqués dans la prédation des ressources naturelles dans cette partie du pays. Au lendemain de la prise du pouvoir par LaurentDésiré Kabila en mai 1997, le Nord-Kivu connaissait déjà une restructuration tendant à s'échapper au contrôle du pouvoir central et ce, pour des raisons évidentes. En effet, les parrains étrangers du chef rebelle Laurent-Désiré Kabila (porte-parole du mouvement rebelle) avaient construit des stratégies pouvant leur permettre de maintenir sous leur emprise l'Est de la RDC en général et le Nord-Kivu en particulier. De ces stratégies, il y a lieu de mentionner le cantonnement des militaires tutsi au Nord-Kivu, l'entretien des groupes armés, la généralisation de l'insécurité, l’instrumentalisation 
ethnique, les assassinats ciblés, les empoisonnements des leaders politiques et militaires, la corruption,...

En ce qui concerne le cantonnement des militaires tutsi à l’Est de la RDC, plusieurs rapports écrits ${ }^{63}$ ont déjà dénoncé cette stratégie depuis l'arrivée au pouvoir du Président Laurent-Désiré Kabila en mai 1997. Avec l'AFDL, les militaires tutsi, y compris les civils, avaient la plénitude de pouvoir dans plusieurs domaines de la vie de l'Etat, particulièrement l'armée et la sécurité. Sachant que le contrôle efficace et effectif d'un pays passe par la main mise sur l'appareil militaire et de sécurité, les militaires tutsi qui avaient accompagné Laurent-Désiré Kabila dans sa conquête militaire s’étaient, depuis la progression militaire, organisés pour assoir leur emprise sur les domaines stratégique de l'Etat. Avec James Kabarebe à la tête de la jeune armée congolaise, Sylvain Buki comme commandant des forces terrestres, l'armée congolaise de l'époque était alors entièrement soumise à la planification et préparation du renforcement du rôle dirigeant des soldats tutsis au sein de l'armée congolaise. Cette configuration de l'armée et du service de sécurité de l'époque avait facilité la mainmise de l’Est du pays par les militaires d'expression rwandaise ayant activement pris part à la rébellion de l'AFDL en 1996-1997.

Sur le plan politique, la présence d'acteurs tels que Bizima Karaha et Déograsias Bugera, pour ne citer que ceux-là, respectivement ministre des affaires étrangères et secrétaire général de l'AFDL sous le régime LaurentDésiré Kabila matérialisait déjà, à cette époque, la tendance hégémonique de la nébuleuse tutsie sur le jeune pouvoir de Laurent-Désiré Kabila. Ce positionnement politique et militaire stratégique des «tutsi congolais » dans les institutions politiques, militaires et sécuritaires au niveau du pouvoir central constituait justement un frein au redéploiement de l'Etat et d'un pouvoir organiseur à l'Est du pays, situation non voulue par les acteurs concernés car susceptibles de nuire aux intérêts spécifiques à protéger à tout prix. Le refus de la relocalisation des militaires tutsi des provinces de l'Est doit se comprendre dans la nécessité des acteurs concernés de rester groupés et réunir leurs forces pour mieux se défendre et se protéger.

Cette reconfiguration du paysage politique et sécuritaire de l'Est du pays avait efficacement aidé et facilité le déclenchement de la guerre du RCD en août 1998, rendue possible par le retournement de quelques officiers militaires congolais déployés à l'Est dont Jean Pierre Ondekane, Joseph Ilondo et les autres. La longue durée de la guerre du RCD (1998-2003) et la partition de facto du pays constituent les facteurs ayant favorisé et précipité la distanciation entre le gouvernement central de Kinshasa et les provinces

\footnotetext{
${ }^{63}$ Lire à ce propos les différents rapports de groupes des experts des Nations Unies (2001,
} 2002, 2008, 2009), les rapports de Grisis Group, de Pole Institue,... 
de l'Est du pays non seulement sur le plan politique et sécuritaire mais aussi économique et social. Durant cette guerre, le Nord-Kivu avait fait l'expérience d’une gouvernance autonome. Si les directives générales et majeures venaient du Rwanda et de l'Ouganda, la matérialisation sur le terrain se faisait par le truchement de certains cadres au sein du mouvement rebelle. Par ailleurs, l'installation de la capitale du mouvement rebelle à Goma avait lourdement contribué à reconfigurer les représentations de certains cadres congolais vers la nécessité d'une gestion autonome de l'Est du pays en détachement total du reste du pays. Seul le «vouloir vivre ensemble » affiché par la population de l'Est du pays et certains cadres du RCD opposés au détachement d'une partie du pays avait pu aider au maintien de l'unité du pays ${ }^{64}$.

Même après la fin officielle de la guerre en 2003, les acteurs politiques et militaires du Nord-Kivu, avaient affiché une forte résistance au rattachement à l'ensemble national. Le maintien de Monsieur Serifuli ${ }^{65}$ comme Gouverneur du Nord-Kivu en 2003 et du Général Obedi Rwibasira comme Commandant de la $8^{\text {ème }}$ Région Militaire du Nord-Kivu avait rendu compliqué le redéploiement effectif du pouvoir central au Nord-Kivu. Le rétablissement de l'autorité étatique dans cette province était perçu, par les acteurs calculateurs, comme la mise en péril de leurs intérêts solidement entretenus dans cette partie riche du pays. Pour ces acteurs, il était essentiel de trouver et mettre en place des mécanismes visant à limiter le redéploiement de l'autorité étatique dans la province en vue de disposer des espaces libres d'exercice de leurs activités de prédation de ressources naturelles congolaises. Il a fallu des tractations au niveau local, national, régional voire international pour que l'espace du Nord-Kivu s’ouvre progressivement aux responsables politiques, administratifs, militaires et sécuritaires venus de Kinshasa pour prendre des postes dans la province. Si, au fil de temps, l'espace politique et administratif du Nord-Kivu s'était ouvert aux cadres et responsables venus de Kinshasa ou d'autres provinces du pays, la passation du pouvoir au niveau de l'armée et de service de sécurité a pris énormément du temps. Il a fallu attendre fin 2004, le replacement du Général Obedi Rwibasira à la tête de la $8{ }^{\text {ème }}$ Région Militaire

\footnotetext{
${ }^{64}$ Les acteurs tels que Jean-Pierre Ondekane, Adolphe Onusumba, le Colonel Ilondo et autres non originaires de l'Est du pays étaient opposés à l’idée de la partition définitive du pays, souhaitant poursuivre la lutte armée jusqu'au renversement du régime de Kinshasa. Cette tendance était renforcée par le soutien de la population locale qui soupçonnait la volonté du Rwanda et de l'Ouganda de maintenir et renforcer leur emprise au pays.

${ }^{65}$ Infirmier anesthésiste, Eugène Serufuli Ngayabaseka rejoint la rébellion du RCD dès le 02 août 1998. Sujet hutu de Rutshuru, Serufuli est désigné à la coordination politique du RCD sous la direction du Pr Vincent de Paul Lunda Bululu et Emile Ilunga. En 2000, à la mort du gouverneur RCD Leonard Kanyamuhanga et contre toute attente, il est désigné à la tête du gouvernorat.
} 
du Nord-Kivu et les élections de 2006 qui sanctionnera la fin du règne de Serufuli à la tête de la province pour que la réunification commence à prendre corps dans cette province de l'Est du pays.

Par ailleurs, le refus de Laurent Nkunda d’intégrer les FARDC et son positionnement au Nord-Kivu (Kilolirwe/Masisi) avec d'autres officiers militaires tutsi, leur action insurrectionnelle à Bukavu en juin 2004, la création du Congrès National pour la Défense du Peuple (CNDP) en 2006 et du Mouvement du 23 mars 2009 (M23),... sont à inscrire dans la stratégie des acteurs concernés à limiter systématiquement l'émergence d'un pouvoir politique et étatique réel et efficace au Nord-Kivu. A chaque tentative du rétablissement de l'autorité de l'Etat, des actions insurrectionnelles ou des attaques armées sont aussitôt construites, planifiées et exécutées en vue de maintenir une situation d'insécurité et d'inorganisation très favorables à la mise en œuvre des activités de prédation de ressources naturelles très convoitées par les multinationales.

En réalité, les acteurs locaux, nationaux et régionaux ne seraient que les intermédiaires d'une chaine économique mafieuse mondiale dont les points finaux sont les industries de consommation et de transformation occidentales et asiatiques. Le problème de gouvernance, de démocratie, de dictature, de protection des minorités, des forces négatives,...souvent évoqués comme facteurs majeurs de la crise congolaise ne sont que de constructions des acteurs impliqués dans la prédation. La problématique de l'accès à la terre et de conflictualité ethnique est bien réelle mais suffisamment instrumentalisée par les acteurs pour de raisons de positionnement ou repositionnement politique. Le maintien de l'insécurité et de l'absence de l'autorité étatique au Nord-Kivu rentrerait dans la logique d’intervention des acteurs étant donné que la prédation de ressources naturelles conviendrait dans une situation d'insécurité et d'inorganisation, situation propice à l'émergence et au développement de l'économie de guerre qui alimente les bandes criminelles.

\section{Difficile contrôle de la province sur les territoires}

Au Nord-Kivu, point n’est besoin de démontrer que le gouvernement provincial exercerait un contrôle plutôt difficile et limité sur l'ensemble des six territoires qui la composent. De la même manière que le gouvernement central exerce un contrôle difficile sur la province du Nord-Kivu, de la même manière qu'il s’observe la difficulté qu'a le gouvernement provincial à exercer un contrôle réel et efficace sur les six territoires de la province. Pour mieux élucider cet état des choses, il convient de recourir à la subdivision administrative et ethnique de la province du Nord-Kivu où l'ethnie Nande, majoritaire dans le Grand-Nord (territoire de Beni et de Lubero), y exerce sa domination et influence sur la situation politique et sécuritaire. Le Petit-Nord 
(territoire de Masisi, Rutshuru, Nyiragongo et Walikale) en dépit de sa multiethnicité se trouverait sous la domination et l'influence des rwandophones (Hutu et Tutsi) qui y exerce un contrôle politique et sécuritaire depuis de longues années. La lutte pour le contrôle et l’exerce du pouvoir au niveau du Nord-Kivu s'effectue, depuis l'avènement des rébellions et des conflits armés récurrents dans la province, entre les Nande et les rwandophones, Hutu et Tutsi réunis dont les clivages internes sont parfois, et d'ailleurs souvent, dépassés au profit de leurs intérêts communs ${ }^{66}$.

La quête de la compréhension des réalités politiques et sécuritaires du Nord-Kivu nécessite de structurer l'analyse sous deux grandes périodes caractérisées chacune par l'exercice du pouvoir politique par l'un de deux groupes ethniques protagonistes dans la province. La première période correspond au règne du Gouverneur Eugène Serufili de l'ethnie Hutu de Rutshuru et la seconde correspond à l'avènement au pouvoir provincial du Gouverneur Julien Paluku de l'ethnie Nande de Lubero. L’arrivée au pouvoir d'Eugène Serufili avait coïncidé avec la tension persistance entre le Rwanda et l'Ouganda avec les affrontements de Kisangani. Après le repositionnement du RCD/K ML dans la ville de Beni, il était donc normal, à cette époque, que le Grand-Nord échappe au contrôle du Gouverneur Serufili basé, lui, à Goma dans le Petit-Nord. De 2000 à 2003, le Grand-Nord était dirigé par le RCD/K ML sous la conduite, dans un premier temps, du Professeur Ernest Wamba dia Wamba, puis d’Antipas Mbusa Nyamwisi.

Mais après la fin officielle de la guerre et l'entrée en fonction du gouvernement de transition le 30 juin 2003, le pays était théoriquement réunifié. En conséquence, le gouvernement central était censé étendre son autorité et son pouvoir sur l'ensemble du territoire national, comme chaque province devrait étendre son autorité sur l'ensemble des districts et territoires qui la composent. Mais à l'intérieur de la province, l'autorité provinciale du Nord-Kivu avait eu du mal à étendre son autorité sur l'ensemble de la province. Basée à Goma, dans le Petit-Nord, l'autorité provinciale ne contrôlait que théoriquement le Grand-Nord qui, sur le plan de faits, échappait effectivement à son contrôle. A la base de cette situation, l'opposition des peuples Nande de voir leur entité dirigée par les rwandophones avec lesquels ils sont en conflits tantôt latent, tantôt manifeste depuis de décennies (Mathe G, 2007). En dépit de la réunification du pays, rares sont les fois où le gouverneur Serufuli se rendait personnellement dans

${ }^{66}$ La nécessité d’obtention d'un espace vital, l'accès aux ressources et aux fonctions publiques, l'intégration parfaite au sein de la société congolaise,... sont autant des facteurs qui amènent et poussent régulièrement les rwandophones de la RDC de faire front commun avec, bien attendu, le support, l'orientation et l'encadrement du Rwanda pour les aider à atteindre cet objectif qui, au final, fait partie intégrante de la stratégie rwandaise d'occupation de la partie orientale de la RDC. 
le Grand-Nord pour donner des directives et orientations de la politique provinciale aux autorités politiques et administratives. Ceci ne pouvait être possible que lorsque le gouverneur devrait accompagner une autorité du gouvernement central telles le Chef de l'Etat, les vice-présidents, les ministres nationaux en mission officielle dans la province. Mais le déplacement du Gouverneur Eugène Serufuli dans les territoires du PetitNord ne souffrait d'aucune restriction protocolaire ou sécuritaire sauf, peutêtre, pour le centre du territoire de Walikale vu son éloignement de la ville de Goma, siège des institutions provinciales.

Les élections de 2006 avaient permis l'arrivée au pouvoir des nouveaux gouverneurs élus en remplacement de ceux nommés par le Président de la République. L'élection du gouverneur au second degré par les députés provinciaux en 2006 avait permis le choix de Julien Paluku ${ }^{67}$. Ce dernier fut l'un des leaders du parti politique RCD/K ML, parti membre de l'Alliance de la Majorité Présidentielle (AMP) du Président Joseph Kabila. L'arrivée à la tête de la province du Nord-Kivu de Julien Paluku, de l'ethnie Nande, ressortissant du Grand-Nord, a été considérée comme un revers pour les rwandophones au pouvoir depuis l'avènement du RCD dans la province. La perte du poste est, pour eux, la perte de l'influence et de la domination qu'ils exerçaient sur les autres ethnies de la province et qui leur donnait avantages politiques et surtout économiques sur les ressources de la province. La lutte politique entre leaders s'oriente ainsi sur le terrain de l'ethnicité ou de l'identité et alimente de crispation entre les groupes ethniques dominants. Parlant de la mobilisation identitaire comme moyen d'expression politique, Cédric Mayrargue et Comi Toulabor (2009) notent ce qui suit: "Pour beaucoup d'observateurs, l'identité serait une de modalité centrale de l'expression politique, une des voies par lesquelles les sociétés négocieraient leur relation à la sphère politique, notamment dans le contexte postérieur aux processus de libéralisation politique et de démocratisation ».

Malgré cette conquête du pouvoir politique par les Nande, sous la houlette de Julien Palulu au gouvernorat de la province du Nord-Kivu, les rwandophones ne s'avouent pas complément vaincu et ne désarment pas pour autant. Ayant perdu le contrôle du pouvoir politique officiel, ils demeurent bien influents et puissants sur le plan militaire et sécuritaire au

${ }^{67}$ Originaire du territoire de Lubero dans le Grand-Nord, Julien Paluku a milité au sein de l'AFDL, dans le RCD et dans le RCD/K-ML où il a exercé plusieurs fonctions politiques. Nommé Administrateur du Territoire Assistant à Lubero, puis Administrateur Titulaire, il est nommé Maire de la ville de Lubero en mars 2003, puis Maire de la ville de Beni en juillet 2005. Il est élu député provincial lors des élections de 2006 dans la circonscription électorale de Butembo. En juillet 2007, il est élu gouverneur de la province du Nord-Kivu, poste qu'il occupe jusqu'à ce jour. Depuis 2012 et juste après les élections présidentielles de 2011, Julien Paluku a créé son propre parti politique dénommé BUREC (Bloc Uni pour la Renaissance et l'Emergence du Congo). 
sein de la Province du Nord-Kivu. Le positionnement stratégique des membres de leur communauté au sein de l’armée congolaise aussi bien au niveau national que provincial constitue encore un gage de sécurité et ouvre encore la voie à l’émergence économique qui constituent des ressources mobilisables dans le processus de conquête de position de domination au sein de la province. Leur positionnement militaire et sécuritaire stratégique leur permet d'avoir toujours droit au chapitre dans les affaires de la province en dépit de la perte actuelle du positionnement politique.

Conscient de cette réalité macabre, l’autorité provinciale actuelle du Nord-Kivu ne serait pas libre de circuler aisément sur l'ensemble de son entité administrative. En tant que Gouverneur, rares sont les déplacements qu'il effectue dans les territoires qui composent le Petit-Nord, à savoir Rutshuru, Masisi, Nyiragongo et Walikale. Ses visites dans ces territoires s'inscrivent souvent dans le cadre d'accompagnement d'une autorité du gouvernement centrale, soit le premier ministre où le président de la République en mission dans cette partie de la province. C'est fut le cas lors de la visite du Président Kabila en septembre 2010 dans le territoire de Masisi ayant abouti à l'interdiction de l'exploitation artisanale des minerais au Kivu. Ce fut également le cas lors de la visite du premier ministre Muzito dans le territoire de Walikale en novembre 2010 pour mesurer les effets de l'interdiction de l'exploitation artisanale des minerais congolais dans les trois provinces de l'Est du pays. Le gouverneur Julien Paluku se rend plus ou moins facilement dans le territoire de Rutshuru où il a de la famille et des relations de longues dates. Mais les visites de ce dernier dans le Grand-Nord ne sont pas à comptabiliser. Qu'il s’agisse de la visite de travail ordinaire, de l'accompagnement de l'autorité nationale ou à titre personnel, Julien Paluku se rend à Beni ${ }^{68}$, Butembo et Lubero comme il le souhaite et sans aucune forme de formalités protocolaires spécifiques. D’abord parce qu’il s’agit des entités qu'il avait eu à diriger personnellement, ensuite parce qu'il s'y sent en sécurité et parfaitement chez lui et, enfin, parce qu'il est membre de l'ethnie Nande qui occupe majoritairement les deux territoires qui constituent le Grand-Nord (territoires de Beni et de Lubero) ${ }^{69}$.

Ce clivage politique entre les Nande et les rwandophones (Hutu et Tutsi) pour la conquête du pouvoir et l'hégémonie dans le Nord-Kivu

${ }^{68}$ Cependant, les visites de Julien Palaku sont tout de même limitées dans le territoire de Beni depuis son départ du RCD/K ML de Mbusa Nyamwisi, originaire de Beni. Pour les Nande de Beni, le Gouverneur devrait démissionner et quitter la majorité présidentielle avec le départ de Mbusa de cette plateforme politique. Mais le Gouverneur du Nord-Kivu avait préféré conserver son poste et pour ce faire, avait créé son propre parti politique actuellement membre de la majorité présidentielle.

${ }^{69}$ Ici, les rivalités inter Nande, entre ceux de Beni et ceux de Lubero, sont momentanément et d'ailleurs souvent occultées au profit de la lutte commune contre les communautés rwandophones de hutu et tutsi réunies. 
alimente l'insécurité qui caractérise la province. Les pratiques de règlements de compte, des empoisonnements, des assassinats politiques, des tueries, des massacres à grande échelle, de la construction et de l'entretien des milices armées, des conflits armés récurrents, des rébellions,... sont autant des conséquences qu'engendre la conflictualité grandissante dans cette partie de la RDC depuis plusieurs décennies maintenant. Pour certains analystes, les événements actuels de Beni constituent une tentative de déstabilisation du Grand-Nord par les acteurs rwandophones du groupe ethnique hutu et tutsi du Petit-Nord en vue d'assurer leur installation sur les espaces encore disponibles.

\section{Porosité des frontières à l'Est du pays}

La mondialisation des échanges économiques, mais aussi culturels et démographiques, est une réalité qui pèse lourdement sur les conditions concrètes d'exercice du pouvoir politique. Les grandes firmes ont des activités à l'échelle planétaire, et le problème de leur « nationalité » est à peu près dépourvu de sens. Or cette mondialisation postule la suppression des entraves à la mobilité des capitaux, suscite des mouvements de personnes à la recherche de l'emploi, contribue à brasser davantage les intérêts. C'est en ces termes que Philippe Braud (2004) s'exprime lorsqu'il évoque le concept de l'Etat déterritorialisé pour analyser la situation des Etats qui voient leurs frontières bousculées par les effets politiques mais surtout économiques de la mondialisation. Dans le monde actuel, les frontières semblent devenir à la fois moins importantes et plus importantes. D'une part, l'évolution économique mondiale amène les Etats à repenser leurs frontières en termes de flexibilité et de coopération. D'autre part, même dans les unions douanières et économiques, les frontières restent la marque la plus visible de la souveraineté d'Etat sur un territoire. Dans cette logique, une gestion efficace des frontières peut être considérée comme un symbole de l'implication du gouvernement dans la protection de sa population (Marenin Otwin, 2010).

Une frontière est la concrétisation d'une opportunité pour un Etat de contribuer au maintien de la sécurité interne en empêchant les menaces extérieures d'atteindre son territoire et sa population. La frontière est, par conséquent, tant une séparation physique entre Etats qu'un processus de contrôle au service de leur sécurité. Néanmoins, les exigences du système mondial économique actuel requièrent un certain degré d'ouverture des frontières. Aussi, les frontières doivent non seulement être exclusives (ne pas laisser entrer les personnes et les biens indésirables) mais également inclusives (faciliter la mobilité des personnes et des biens). Il est donc important pour un Etat de trouver l'équilibre entre le besoin de mobilité et le besoin de contrôle entre «des portes et des murs ». Une frontière 
complètement fermée n'est pas la solution. Au contraire, elle risquerait de créer davantage un environnement propice pour les activités illicites (Jihan Seniora et Cédric Poitevin, 2010).

En effet, certaines régions frontalières peuvent devenir des zones de refuges pour des malfaiteurs et des lieux de criminalité et de trafic où les déplacements de trafiquants et criminels sont éventuellement facilités par les complicités ethniques des populations des régions frontalières... Les routes de trafic contournent les points de contrôle à la frontière, utilisant les parties de territoires où les solidarités entre populations locales priment. Cette réalité est beaucoup plus observable dans l'Afrique de l'Ouest, mais aussi et, surtout actuellement, en Afrique centrale, particulièrement dans la région des Grands-Lacs. Tel est le cas actuel de la RDC avec ses voisins de l'Est où la solidarité ethnique entre les populations locales d'expression rwandaise vivant le long de la frontière est devenue très forte et facilite le déroulement d'activités criminelles souvent protégées par cette solidarité. La gestion frontalière de l'espace frontalier depuis le nord-est jusqu'au sud-est, du territoire d'Aru jusqu'à Kalemie en passant par Bunia, Kasindi, Beni, Butembo, Lubero, Rutshuru, Bunagana, Goma, Bukavu, Uvira, Fizi appelle un contrôle efficace et ordonné.

Mais notre analyse se focalise sur la province du Nord-Kivu dont les frontières avec les pays de l'Est part de Kasindi jusqu'à Goma en passant par Beni, Butembo, Lubero, Rutshuru et Bunagana. En effet, ces frontières entre la RDC d'un côté et le Rwanda et l'Ouganda de l'autre, distant de plus ou moins 400 kilomètres, disposent de trois points frontaliers officiels qui sont le poste de Kasindi en territoire de Beni, le poste de Bunagana en territoire de Rutshuru et le poste de Goma (grande et petite barrière) où les personnes et les biens doivent traverser officiellement. Mais en dehors de ces postes frontaliers officiellement reconnus, existent de multitude points de passage servant de la contrebande des produits illicites tels que les armes légères, les produits miniers, les bois, les personnes sous mandat d'arrêt international,... La route reliant Beni et Goma se trouvant le long de la frontière et à seulement 2 à 10 kilomètres dans certains endroits facilite le passage incognito d'hommes et des biens à l'insu des structures officielles. L'analyse montre que de plus en plus de personnes et des biens traversent les frontières par des voies illicites que licites où les taxes et autres prélèvement de l'Etat coûtent chers.

L’une des caractéristiques essentielle de la conflictualité du NordKivu depuis le déclenchement des conflits armés est la porosité régulièrement observée le long des frontières entre la RDC et ses pays voisins. La porosité des frontières est une conséquence voulue par les acteurs impliqués afin de faire passer aisément les ressources naturelles illégalement exploitées vers les pays voisins, lieux de centralisation, d'entreposage, de 
conditionnement avant de prendre la direction de l'océan indien pour leur destination finale, les pays occidentaux et asiatiques. Pour le cas du NordKivu, Kampala et Kigali constituent la plaque tournante de centralisation des ressources naturelles congolaises qui traversent illicitement par les frontières officielles et non officielles. En effet, l'observation rigoureuse de la porosité des frontières de l'Est congolais témoigne que cette perméabilité se trouve renforcer par la présence, l'attitude et le comportement des agents commis à la tâche de surveillance des frontières de la RDC. Depuis le déclenchement des rébellions de l'AFDL mais surtout celle du RDC, les frontières congolaises avec ses voisins fonctionnent à deux vitesses différentes.

D’une part, le Rwanda et l'Ouganda assurent un contrôle régulier, normal et classique sur tous les points frontaliers avec la RDC. Les agents commis à la surveillance et à la garde des frontières exécutent leurs tâches de façon professionnelle, sans pression supplémentaire et filtre les personnes et les biens susceptibles de pénétrer sur les territoires de leurs Etats. Cependant, les cas isolés de fraude de manquent pas, à l'instar de plusieurs pays africains voire du monde. D’autre part, le contrôle des frontières côté congolais semble remplir toutes les exigences d’irrégularité, de corruption, d'inattention, de complaisance et de subordination. La situation parait bien voulue, connue et favorisée par les acteurs impliqués dans la crise congolaise. Le désordre, la corruption généralisée, le trafic d’influence, le favoritisme, le clientélisme,...ont cessé d'être l'exception pour devenir la règle. Il est curieux de se rendre compte, au niveau des frontières congolaises avec les pays de l'Est, qu'une bonne partie d'agents, occupant des postes stratégiques, est d'expression rwandophone, en dépit du fait que cette partie du territoire regorge également les autochtones d'expression rwandophone ${ }^{70}$.

Dans cette configuration, le contrôle se fait de part et d'autres des frontières par les agents rwandophones ou leurs acolytes pour la facilitation du trafic illicite au profit du Rwanda et de l'Ouganda. Les quelques tentatives de dénonciation sont soit camouflées ou réprimandées par les agents haut gradés implicitement impliqués dans la manœuvre, soit classées sans suite ou rangées dans les oubliettes. D’autres cas sont réglés sur place, au niveau des frontières, par la négociation ou par la force souvent au bénéfice des rwandophones encore influents dans le Petit-Nord. A titre d'exemple, deux sujets rwandais, non en ordre pour traverser la frontière vers Goma à la petite barrière, ont été empêchés par un agent congolais commis à la surveillance de la frontière. Après négociation, l'agent congolais était catégorique et exigeait que les deux sujets rwandais puissent retraverser la frontière et régulariser leur situation avant d'entrée en RDC. Mais 15

\footnotetext{
${ }^{70}$ Observations faites par nous-mêmes au niveau de la grande et de la petite barrière à Goma et à Bunagana dans le poste frontalier du territoire de Rutshuru.
} 
minutes plus tard, une camionnette remplie des militaires tutsi est arrivée sur le lieu pour, non seulement libérer les deux sujets rwandais mais aussi tabasser et embarquer l'agent congolais pour une destination inconnue et ce, sous le regard silencieux de tous les autres agents congolais travaillant à la petite barrière de Goma ${ }^{71}$.

Ceci est un cas parmi tant d'autres qui attestent que les frontières congolaises sont non seulement perméables mais aussi contrôlées par les personnes impliquées dans la poursuite de la prédation des ressources au Nord-Kivu. Elles maintiennent cette perméabilité pour le trafic illicite des ressources naturelles congolaises et s'emploient à résister à toute tentative de rétablissement de l'ordre, du contrôle réel, de la surveillance et de l'autorité de l'Etat le long des frontières entre la RDC et le Rwanda et l'Ouganda. La mondialisation aidant, la disponibilité des capitaux étrangers en quête des ressources naturelles trouvables en quantité importante en RDC, la résistance des congolais à la prédation de leurs ressources naturelles par la globalisation, la complicité de certains acteurs nationaux et locaux,... sont là des facteurs qui attestent que la paix à l'Est du pays et la stabilité transfrontière risque de demeurer encore longtemps des concepts théoriques sans matérialité dans l'immédiat.

\section{Conclusion}

Les conflits armés qui sévissent au Nord-Kivu depuis les années 1990 impactent négativement sur la capacité d'intervention de l'Etat congolais non seulement au niveau central mais surtout au niveau du pouvoir provincial. Bien que la défense nationale, par le truchement de l'armée, soit la prérogative première du pouvoir central, ce sont les dirigeants de l'entité concernée qui subissent en premier les assauts et les conséquences directes et indirectes de la violence armée dans la région. L’implication de divers acteurs (locaux, nationaux, régionaux et internationaux) dans la poudrière au Nord-Kivu en quête d'intérêts particuliers a favorisé l'émergence et l'enracinement d'une économie de guerre protégée par les acteurs impliqués.

La persistance des conflits armés a reconfiguré l'environnement socio-économique de la province du Nord-Kivu qui s’observe à travers trois principaux axes. D’abord la mise en place d'une économie de guerre favorisée par l'autofinancement de la guerre grâce à l'exploitation illicite de ressources naturelles de la province et leur insertion dans l'économie mondialisée. Ensuite par le truchement de la militarisation de l’économie locale où les opérateurs économiques du Nord-Kivu travaillent en parfaite

\footnotetext{
${ }^{71}$ L'incident avait eu lieu en date du 25 octobre 2010 à la petite barrière de Goma. L'agent tabassé a été acheminé à l'Etat-major militaire de Goma et jeté au cachot sans jugement ni explication sur son attitude et comportement face aux sujets rwandais qui, eux, avaient rejoint Goma en dépit de leur irrégularité.
} 
collaboration avec les acteurs armés pour la sécurisation de leurs activités. Enfin, l'extraversion de l'économie locale vers les pays de l'Afrique de l'Est et de l’Océan indien aboutit à la distanciation du Nord-Kivu des provinces de l’Ouest du pays et de Kinshasa.

Cette reconfiguration de l'environnement socio-économique du Nord-Kivu, fruit des acteurs armés et économiques, a produit des effets néfastes sur l'environnement politique et sécuritaire de la province. D’abord le constat d'un contrôle difficile du pouvoir central sur la province du NordKivu à travers le refus du redéploiement des soldats tutsi dans d'autres provinces du pays, l'activisme et la prolifération des groupes armés, les assassinats, les empoisonnements, les enlèvements,... Ensuite le difficile contrôle de gouvernement provincial sur les six territoires que compte la province où l'autorité provinciale n'y arrive pas à exercer un contrôle réel et efficace en fonction de son appartenance ethnique (lutte entre le Grand et le Petit-Nord). Enfin, la porosité des frontières entre la province du Nord-Kivu et les pays voisins de l'Est, le Rwanda et l'Ouganda en particulier favorise la poursuite de la contrebande et le passage incognito des ressources naturelles illicitement exploitées dans la province.

\section{References:}

1. Braud, P. (2004), Penser l'Etat, Seuil, Paris.

2. Collier, P., et Hoaffler. (2013) A, « On economic causes of civil war », Oxford Economic Paper, Volume 50.

3. Doyle, M., and Sambanis, N. (2000), « International peacebuilding : a theoritical and quantitative analysis », American Political Science Review, Volume 94, Number 4.

4. Fearonj, D., and Laitin, DD. (1997), « Ethnicity insurgence and civil war, Americal polical scicne review ».

5. Hugo, P. (Mars 2009), «Le rôle des ressources naturelles dans les conflits en Afrique », La découverte $n^{\circ} 134$.

6. Jacquemot, J. (2009), «L'économie des conflits dans le Congo Oriental », Hérodote $n^{\circ} 134$, La Découverte, Paris.

7. Jihan Seniora et Cédric Poitevin (Mars 2010), Armes légères : gestion des frontières terrestres et trafic illicite, Rapport du GRIP.

8. Jourde, C. (2009), «Les grilles d'Analyse de la politique africaine : la problématique de l'Etat », in Mamadou Gazibo et Céline Thiriot, Le politique en Afrique. Etat des débats et pistes de recherche, Paris, Karthala.

9. Le Billon, P. (2003), « Matières premières, violences et conflits », Revue tiers Monde $n^{\circ} 174$.

10. Marenin Otwin. (2010), Challenges for integrated border management in the European Union, Geneva Center for the 
Democratic Control for Armed Forces Occasional, Paper n¹7, 2010. Cité par Jihan Seniora et Cédric Poitevin, Armes légères : gestion des frontières terrestres et trafic illicite, Rapport du GRIP.

11. Mathe, G. (2007), « Conflits locaux, guerre régionale : anatomie des crises politiques au Nord-Kivu », in Maindo Manga Ngonga (Dir.), Des conflits locaux à la guerre régionale en Afrique Centrale. Le Congo-Kinshasa oriental 1996-2007, l'Harmattan, Paris.

12. Mayrargue, C., et Toulabor, C. (2009), «L'expression et la participation politique en Afrique » dans Mamadou Gazibo et Céline Thiriot, Le politique en Afrique. Etat des lieux et pistes de recherche, Paris, Karthala.

13. Mbembe, A. (2000), De la post-colonie. Essai sur l'imagination politique dans l'Afrique contemporaine, Paris, Karthala.

14. Pourtier, R., (2009), "Le Kivu dans la guerre : Acteurs et enjeux », EchoGéo, Sur le vif, 2009, p. 23, mis en ligne le 21 janvier 2009.

15. Preyssinet-Dominjon, J., Méthodes de recherches en sciences sociales, Paris, Montchrestien, E.J.A, 1997.

16. Rapport du groupe d'expert de l'ONU sur l'exploitation illicite de ressources naturelles en République Démocratique du Congo, Rapport de 2008.

17. Reno, W. (1999), « La privatisation de la souveraineté et la survie des Etats faibles ", in B. Hibou (éd), La privatisation des Etats, Paris, Karthala.

18. Reynal-Querol. (2002), «Ethnicity, political system and civil war », Journal of Conflicts Resolution Volume 41, Number 1. 